\title{
Transfer of various polychlorinated dibenzo- $p$-dioxins and dibenzofurans (PCDDs and PCDFs) via placenta and through milk in a marmoset monkey
}

Hanspaul Hagenmaier 1 , Thomas Wiesmüller 1 , Georg Golor ${ }^{2}$, Ralf Krowke ${ }^{2}$, Hans Helge ${ }^{3}$, and Diether Neubert ${ }^{2}$ Arch Toxicol (1991) 64: 601-615

Several rather misleading printing errors were overloaded in Table 5 (p. 607). Lines 18 to 22 should read:

$\begin{array}{lllll}\text { Line 18: } & 20 & 2378-\mathrm{T} 4 \mathrm{CDF} & <10 & 31 \pm 32 \% \\ \text { Line 19: } & 48 & 12468-P 5 \mathrm{CDF} & <4 & <10 \\ \text { Line 20: } & 29 & 23479-P 5 \mathrm{CDF} & <4 & <10 \\ \text { Line 21: } & 58 & 13479 / . .-P 5 \mathrm{CDF} & 5 \pm 40 \% & <10 \\ \text { Line 22: } & 48 & 12478-P 5 \mathrm{CDF} & 7 \pm 29 \% & <10\end{array}$

